\title{
Physiotherapy for large airway collapse: an ABC approach
}

\author{
Lizzie J.F. Grillo (1) ${ }^{1,2}$, Georgie M. Housley ${ }^{1}$, Sidhu Gangadharan $\mathbb{1}^{3}$, Adnan Majid ${ }^{3}$ and James H. Hull (1) ${ }^{1,2,4}$
}

${ }^{1}$ Royal Brompton and Harefield Hospitals, London, UK. ${ }^{2}$ National Heart and Lung Institute, Imperial College, London, UK. ${ }^{3}$ Beth Israel Deaconess Medical Center, Harvard Medical School, Boston, MA, USA. ${ }^{4}$ Institute of Sport, Exercise and Health, UCL, London, UK.

Corresponding author: Lizzie J.F. Grillo (l.grillo@rbht.nhs.uk)

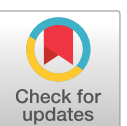

This version is distributed under the terms of the Creative Commons Attribution Non-Commercial Licence 4.0. For commercial reproduction rights and permissions contact permissions@ersnet.org

This article has supplementary material available from openres.ersjournals.com

Received: 17 Aug 2021 Accepted: 17 Oct 202

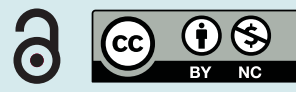

Shareable abstract (@ERSpublications)

Large airway collapse causes debilitating symptoms of cough, retained secretions, exercise intolerance and breathlessness. This review describes the key role that physiotherapy plays in the conservative management of this condition. https://bit.ly/3pADTUp

Cite this article as: Grillo LJF, Housley GM, Gangadharan S, et al. Physiotherapy for large airway collapse: an ABC approach. ERJ Open Res 2022; 8: 00510-2021 [DOI: 10.1183/23120541.00510-2021].

\section{Abstract}

Large airway collapse (LAC) describes the phenomenon of excessive, abnormal, inward movement of the large airways (i.e. trachea and/or main bronchi and/or bronchus intermedius) occurring during the expiratory phase of the respiratory cycle. It is an increasingly well-recognised problem and a prevalent comorbidity in other chronic respiratory conditions (e.g. COPD and asthma). LAC is associated with pervasive respiratory features such as a barking cough, exertional dyspnoea and an increased propensity to lower respiratory tract infection. These symptoms are unpleasant, and patients are often limited in their daily life and their function. The pathophysiology of this condition impairs airway clearance and can cause breathlessness and exercise intolerance, due to a loss of airway patency during expiratory flow. Dysfunctional adaptations to breathing and coughing may further amplify symptoms.

This article provides, for the first time, clinically focused physiotherapeutic intervention advice based on our understanding of the pathophysiology of LAC, to support conservative management. It uses the available evidence from LAC, transferable evidence from other conditions and knowledge based on clinical experience. It proposes a practical "ABC model” to ensure physiotherapy assessment and treatments are centred around optimising three key clinical areas: Airways, including airway clearance and cough; Breathing, including breathlessness and breathing pattern; and Capacity for exercise, including an assessment of functional exercise ability.

\section{Introduction}

Large airway collapse (LAC) describes the phenomenon of excessive, abnormal inward movement of the large airways (i.e. trachea and/or main bronchi and/or bronchus intermedius), occurring during the expiratory phase of the respiratory cycle [1]. Several terms have been used to describe the entities causing LAC, including tracheobronchomalacia (TBM), defined as a weakness of the large airway wall, due to softening of the cartilaginous tracheal wall, or excessive dynamic airway collapse (EDAC), when there is evidence of significant invagination of the posterior muscular tracheal membrane [2]. Regardless of the terminology employed, LAC is increasingly recognised as being associated with debilitating respiratory symptoms, including a barking (or "seal-like” or "honking”) cough, impaired airway clearance leading to respiratory tract infection and exertional dyspnoea [3, 4]. Moreover, the symptoms caused by LAC are pervasive and often result in functional impairment and impaired quality of life [4].

The precise prevalence of LAC remains unclear, influenced by debate regarding diagnostic criteria [5]. However, in a series of 2150 patients undergoing bronchoscopy, 5\% were found to have some form of LAC, with 53\% of these having a concurrent diagnosis of chronic bronchitis [6]. Other studies employing computed tomography (CT) imaging to affirm a diagnosis indicate that almost a third of patients with COPD may have LAC [7]. Additionally, LAC may be present in around $41 \%$ of asthmatic patients undergoing bronchoscopy, with $\sim 30 \%$ classified as EDAC [8]. The prevalence of both TBM and EDAC was directly related to age, sex (female) and asthma severity. 
Following diagnosis, treatments for LAC focus on attempting to "splint" open the large airways. Techniques utilised include the use of positive airway pressure to provide "pneumatic airway splinting" with either continuous positive airway pressure support (CPAP) or noninvasive ventilation (NIV) [9-11] or, in more severe cases, with direct mechanical splinting, for example, with stent placement [4] or a more definitive surgical intervention, termed tracheobronchoplasty (TBP). The latter involves a robotic or, most frequently, an open right posterolateral thoracotomy, typically with application of a proline mesh to stabilise the posterior wall of the trachea, main bronchi and bronchus intermedius [12].

Despite progress in surgical options, several of the key respiratory abnormalities encountered in patients with LAC fall within respiratory physiotherapy expertise. To the best of our knowledge, there are currently no practical guidelines for physiotherapy practitioners in this field and a paucity of evidence on the most effective approach to interventions. The aim of this article is therefore to provide a review of this area, focusing on how physiotherapy can be used to assess and optimise three key clinical features of LAC: Airways including airway clearance and cough, Breathing including breathlessness and breathing pattern, and Capacity for exercise, described as an "ABC model”. The overall aim is to provide pragmatic and clinically focused intervention advice, built on our current understanding of the pathophysiology of LAC.

\section{Understanding the pathophysiology of LAC}

In healthy individuals, there is a degree of inward movement of the large airways (termed dynamic collapse) during exhalation. This movement is further exaggerated during forced expiration and coughing, as a "normal" physiological process, and is an essential component of the secretion clearance mechanisms. Some clinicians classify $>50 \%$ reduction in luminal cross-sectional area of the trachea on expiration as abnormal; however, this degree of collapse is often seen in asymptomatic healthy young individuals and thus $>70 \%$ collapse on bronchoscopy or expiratory CT may be a more pragmatic diagnostic threshold [13]. Regardless, there is often an apparent disconnect between the degree of collapse seen and the symptomatic clinical burden which may be due to the contribution from coexisting conditions (figure 1) [14, 15].

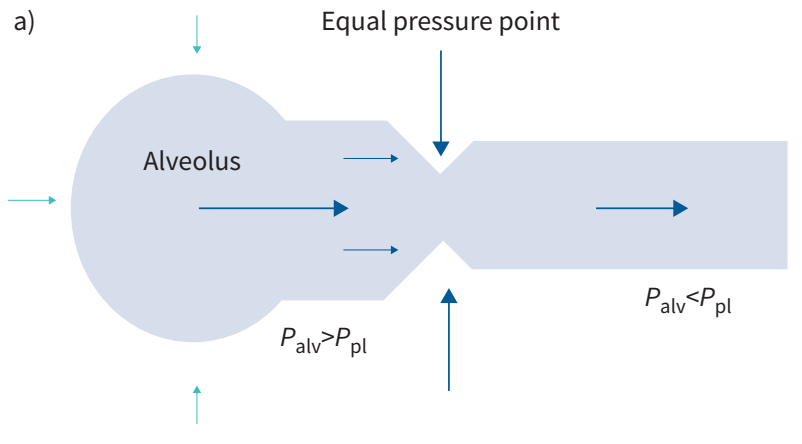

Flow-limiting segment lengthens proximally limiting impact of two phased gas liquid flow

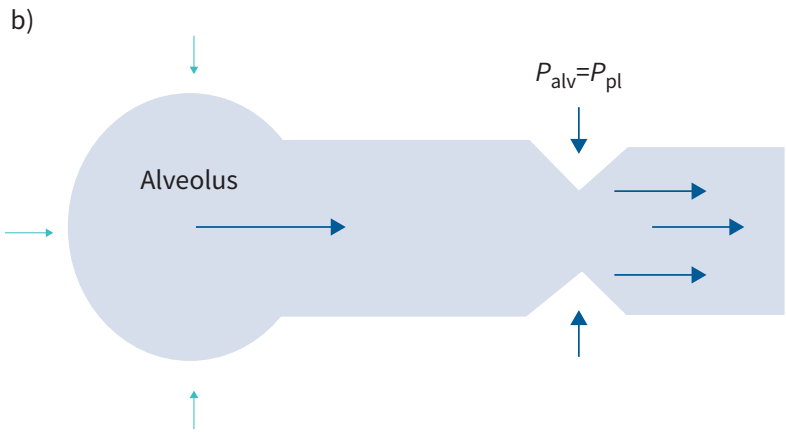

Equal pressure point in a normal airway, stays proximal and facilitates movement of secretions

FIGURE 1 Flow-limiting segment in large airway collapse (LAC). a) Airway with LAC. The flow-limiting segment is limiting the expiratory airflow and/or creating unwanted turbulent airflow due to the invagination of the airway influenced by the equal pressure point theory and wave speed theory. $P_{\mathrm{pl}}$ : pleural pressure; $P_{\text {alv: }}$ : alveolar pressure. b) Normal airway. The equal pressure point (EPP) is kept proximal and promotes movement of expiratory airflow to enhance sputum clearance. 
Models to understand pathophysiology of LAC

Equal pressure point theory

Alveolar pressure $\left(P_{\text {alv }}\right)$ is the driving pressure that causes gas to flow through the airways on expiration.

This driving pressure depends on lung volume, which is influenced by pleural pressure $\left(P_{\mathrm{pl}}\right)$ and elastic recoil of the tissues $\left(P_{\mathrm{st}}\right)$ (i.e. $\left.P_{\mathrm{alv}}=P_{\mathrm{pl}}+P_{\mathrm{st}}\right)$. Pressure decreases as air flows from the smaller or more distal airways to the larger proximal airways. During forced expiration, an equal pressure point (EPP) occurs where extraluminal and intraluminal pressures become equal [16]; this point can then be considered to divide the airway into a distal (upstream) and proximal (downstream) segment. Distally, in the upstream segment, the transpulmonary pressure is positive $\left(P_{\mathrm{alv}}>P_{\mathrm{pl}}\right)$ and thus keeps the airways open. In contrast, more proximally (i.e. downstream or centrally) from the EPP, the airways are compressed because the transpulmonary pressure is negative within the airways $\left(P_{\mathrm{alv}}<P_{\mathrm{pl}}\right)$. In the normal healthy lung, the compressed segment sits within the central airway and resistance to total airway collapse is provided by the cartilage and smooth muscle [17]. In clinical diseases associated with LAC, however, this stability is lost or there are changes in the length or position of the flow-limited segment (FLS) within the airway tree making it more prone to collapse [14].

During forced manoeuvres, for example, as lung volume decreases during the expiratory phase of respiration (i.e. moving from total lung capacity to residual volume), the EPP moves distally and the FLS lengthens but stays in the larger proximal airways. Lung elastic recoil, small airway resistance, smooth muscle tone and airway compliance are major factors influencing where the EPP will be located (see figure 2) [15].

\section{Wave speed theory}

An additional explanation to describe the pathophysiology in LAC is based on "wave speed theory". This suggests that the development of the FLS depends on the speed at which moving air propagates pressure waves. These waves are created from the interaction of recoil force of elastic airway walls and the inertial force of the flowing gas [18]. Expiratory flow limitation occurs when the airflow velocity equals the speed of propagation of pressure wave. This is called the choke point and occurs where the airway pressure does not change with an increase in driving pressure $\left(P_{\text {alv }}\right)$ [19]. Critically the loss of airway calibre within the airway lumen will disrupt the flow, increasing turbulence within the airway, potentially limiting clearance.

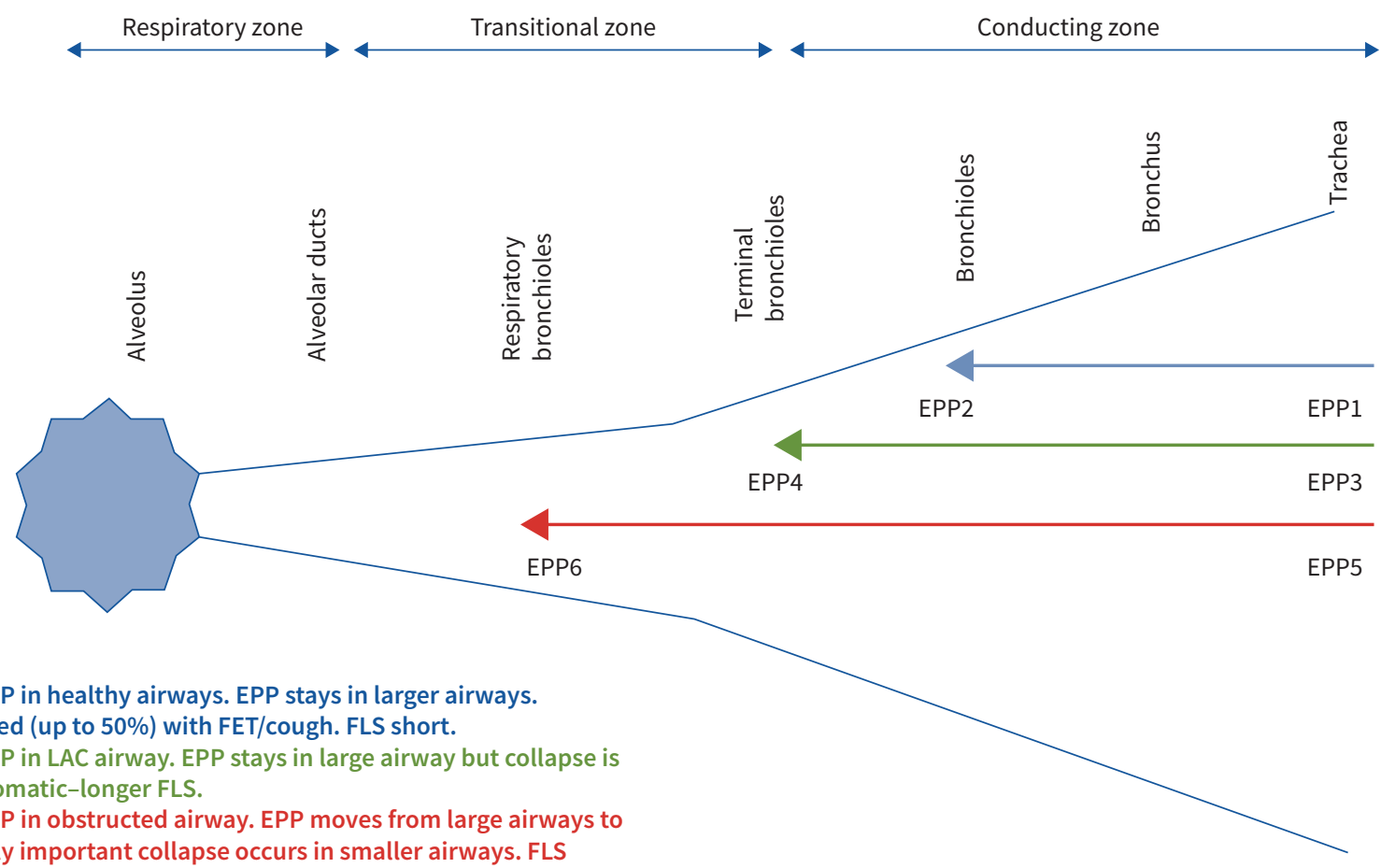

EPP1-2: Movement of EPP in healthy airways. EPP stays in larger airways Tracheal collapse is limited (up to 50\%) with FET/cough. FLS short. EPP3-4: Movement of EPP in LAC airway. EPP stays in large airway but collapse is greater and more symptomatic-longer FLS.

EPP5-6: Movement of EPP in obstructed airway. EPP moves from large airways to smaller airways. Clinically important collapse occurs in smaller airways. FLS lengthens further.

FIGURE 2 Dynamic movements of equal pressure points in different examples of airway physiology. EPP: equal pressure point; FET: forced expiration technique; FLS: flow-limited segment. 
TABLE 1 Subjective and objective physiotherapy assessment for a patient with large airway collapse (LAC)

Subjective: Patient's description/report

\section{A: Airways}

NASAL SYMPTOMS

Blocked or runny nose

Sinus pain

Post-nasal drip

Altered sense of smell

AIRWAY CLEARANCE

Technique completed

Frequency and length of Rx

Efficacy

$\%$ adherence

B: Breathing

BREATHLESSNESS

Symptoms

Triggers

Recovery techniques

COUGH

Triggers

SPUTUM

24-h volume

VOICE/UA
Effectiveness/ease of clearance

Dry/rattling/productive

Feeling of airway closure

Colour/consistency

Voice changes, e.g. husky/strained/lost voice Work of breathing

Closure/discomfort in throat

BREATHING PATTERN

Awareness of breathing pattern

$\mathrm{S}_{\mathrm{pO}_{2}}$

Objective: Physiotherapist's observations/measurements

NASAL SYMPTOMS

Quality of secretions

Throat clearing

AIRWAY CLEARANCE

Observed technique

Impact of airway closure on clearance Microbiology

Observed effectiveness

\section{COUGH} SPUTUM
Nature/sound,

e.g. barking/honking sound

Viscosity/colour of sputum

Upper limb fixation

Accessory muscle use

Auscultation

VOICE/UA

Quality

BREATHING PATTERN

Completion of BPAT score
Audible upper airway sounds

-Upper chest versus lower chest

-Nose-mouth breathing

-Flow/air hunger/RR/rhythm

\section{C: Capacity for exercise}

EXERCISE ABILITY

Frequency of exercise

Intensity of exercise

Time spent on exercise

Type of exercise

Symptoms with exercise

-cough/airway closure

General physical activity levels

Barriers to exercise

Use of oxygen/NIV/CPAP for exercise

Other Ax

SLEEP

Quality/duration

Epworth sleep score

NIV/CPAP history +/- use

\section{EXERCISE TESTING}

6MWT

1 min STS

Ax with and without CPAP/NIV

Cough/SOB $/ \mathrm{WOB} / \mathrm{S}_{\mathrm{pO}_{2}}$

Breathing pattern changes during exercise

UA SYMPTOMS

Observed adaptations to breathing

Audible airway collapse

\section{OUTCOME MEASURES}

VAS: ease of clearance/airway closure

Dyspnoea-12

BPAT

MRC grading

Leicester cough questionnaire (LCQ)

Rx: treatment; $\mathrm{S}_{\mathrm{PO}_{2}}$ : oxygen saturation measured by pulse oximetry; Ax: assessment; UA: upper airway; NIV: noninvasive ventilation; CPAP: continuous positive airways pressure; RR: respiratory rate; SOB: shortness of breath; 6MWT: 6-min walk test; 1 min STS: 1-min sit-to-stand test; BPAT: breathing pattern assessment tool; WOB: work of breathing; VAS: visual analogue scale; MRC: Medical Research Council.

Approach to physiotherapy assessment in LAC

An initial physiotherapy assessment for LAC should include a comprehensive evaluation of the patient's history, focusing on how symptoms impact on functional capacity and quality of life. It is preferable to use patient-reported outcome measures (e.g. Visual Analogue Scale and questionnaire/other scores, e.g. Medical Research Council (MRC) Dyspnoea Scale), where possible, to characterise the level of impairment and inform subsequent disease surveillance and outcome monitoring. A full assessment will typically require 45 minutes to 1 hour, and in some cases may require additional assessments (see table 1).

The ABC model of physiotherapy assessment

A: Airways: airway clearance and cough

Pathophysiology in relation to $L A C$

It is well recognised that impaired airway clearance leads to sputum impaction, leading to localised pulmonary airspace, bronchial wall inflammation and associated damage and suppuration [20]. If untreated this can lead to bronchiectasis and recurrent or persistent pulmonary sepsis contributing to the "vicious circle" hypothesis [21]. 
In order to move secretions high enough for adequate clearance, the proximal airways depend on aerodynamic changes within the airway to facilitate secretion movement [22]. Specifically, to clear secretions, a high expiratory airflow is required to produce a "two-phase air-liquid flow" effect by which energy is transferred from the gas (air) to the liquid (secretions). This results in turbulence and creation of a shearing effect on secretions, aiding the expectoration of sputum [23]. Effective airway clearance aims to utilise the EPP and specifically aims to maintain its proximal location, thereby acting to enhance airflow and ultimately secretion movement.

The pathophysiology of LAC impedes the normal airway clearance process by moving the EPP distally, causing airway compression as the FLS lengthens [14]. This is amplified during manoeuvres that cause rapid changes in intrathoracic pressure or suddenly increase expiratory airflow (e.g. coughing) [19]. Accordingly, the faster the airflow, the greater the collapse, further limiting the proximal movement of secretions. In addition, the loss of proximal airway calibre in LAC creates increased generation of pressure waves working against any shearing forces created by expiratory airflow manoeuvres [3]. This may create choke points analogous to the EPP, thus further limiting effective clearance.

All airway clearance techniques for LAC should consider augmentation of ventilation and hydration of airways [24] (described below). Additionally, patients need to be taught how to best promote effective expiratory airflow, while maintaining airway patency.

\section{Assessment of airway clearance and cough}

Assessment for airway clearance in LAC should follow a similar process to any airway clearance assessment by including a detailed subjective and objective assessment [25, 26]. However the impact of LAC on cough effectiveness means it should start with a detailed discussion about the nature of the cough and its effectiveness including establishing how "effective" a cough is. In particular, the clinician should establish if dynamic collapse is impacting on the ability to clear secretions [4]. Commonly used approaches include the use of subjective patient-reported outcome measures, as well as auscultation to evaluate cough effectiveness [27]. We recommend that therapists listen to the quality of the cough to determine how much any collapse (usually a clear sounding low pitched noise) is impacting on cough efficacy. Classically, significant airway collapse is variably described as a honking, barking or seal-like cough (linked audio file). Additionally, patients should be asked for details on their sputum including its colour, consistency and viscosity.

Advanced assessment should evaluate contribution to cough from upper airway dysfunction (e.g. laryngeal hypersensitivity). For example, factors that may impact on cough include reflux and/or post-nasal drip, both of which can cause upper airway irritation and increased cough frequency, such that airway clearance is impaired $[27,28]$. Additionally, evaluation of breathing pattern, especially during airway clearance, such as gasping, breath holding, upper chest movement and reduced expiratory time are important as these can impact on the ability to breathe in [29], which in turn may limit the effectiveness of forced expiratory manoeuvres.

\section{Airway clearance treatment}

It is standard practice for physiotherapists to educate patients about their respiratory disease and teach an airway clearance technique (ACT), for example, in those with chronic sputum production and evidence of radiological bronchiectasis [30] and cystic fibrosis [25]. However, there is limited evidence defining the optimum ACT in bronchiectasis or other suppurative conditions [26]; however, patient preference, effectiveness of technique and airway physiology may direct choice [22]. The final management plan should be personalised to the individual patient to ensure it is feasible and thus facilitates good adherence [31]. The guidance in this review for patients with LAC, therefore, arises from a synthesis of clinical expertise, experience and transferrable knowledge from other conditions (see table 2).

\section{Recommendations for ACT management}

All patients with excessive sputum production should be taught to complete regular ACT daily by a specialist physiotherapist with a review at 3 months, to optimise technique [26]. Those without regular sputum should be taught an ACT for use during an exacerbation. All patients with exacerbations should complete ACT twice a day minimally and complete a session of ACT for 15 minutes or until two forced expiration techniques (FETs) are clear of sputum [32] (see below). Patients reporting a persistent deterioration in their condition either as an outpatient or inpatient should be referred for a physiotherapy review as part of ongoing disease surveillance and optimisation to ensure ACT is optimised [30]. This advice, which is pertinent to patients with bronchiectasis, can be extrapolated to those with recurrent infections and LAC in the absence of condition-specific advice. 


\begin{tabular}{|c|c|c|c|}
\hline ACT & Physiological principles & Evidence & Pragmatic approach in LAC \\
\hline ACBT & $\begin{array}{l}\text { Flexible three phase cyclical technique of breathing } \\
\text { control, TEE and FET } \\
\text { Enhances tidal volumes, collateral ventilation and } \\
\text { expiratory airflow } \\
\text { Modification for use in LAC to ensure FET is optimised }\end{array}$ & $\begin{array}{l}\text { Physiological } \\
\text { background [32] } \\
\text { Bronchiectasis [34] } \\
\text { Cystic fibrosis [25] }\end{array}$ & $\begin{array}{l}\text { No direct evidence } \\
\text { - Clinical experience in LAC demonstrates that caution } \\
\text { may be required in FET to ensure balance of airway } \\
\text { calibre with creation of equal pressure points }\end{array}$ \\
\hline PEP & $\begin{array}{l}\text { A flow-regulated technique that has three effects: to } \\
\text { increase lung volume (functional residual capacity } \\
\text { and tidal volume }\left(V_{T}\right) \text { ), to reduce hyperinflation and } \\
\text { to improve airway clearance. Positive pressure is then } \\
\text { achieved by augmenting expiratory flow against this } \\
\text { resistance }\end{array}$ & $\begin{array}{l}\text { Physiological } \\
\text { background [35] } \\
\text { COPD [39] } \\
\text { Cystic fibrosis [38] } \\
\text { TBM (improve } \\
\quad \text { expiratory airflow) } \\
\text { [40] }\end{array}$ & $\begin{array}{l}\text { No direct evidence } \\
\text { - Use in LAC to reduce airway closure during } \\
\text { expiration and modify FET } \\
\text { - Caution to prevent airway collapse but not to cause } \\
\text { limitation to expiratory airflow }\end{array}$ \\
\hline OPEP & $\begin{array}{l}\text { PEP is applied by blowing out against a variable } \\
\text { resistance that produces an oscillation in flow } \\
\text { Individuals breathe out to expiratory reserve volume } \\
\text { (ERV) enabling a modulation of both pressure } \\
\text { and flow }\end{array}$ & $\begin{array}{l}\text { Physiological } \\
\text { background [22] } \\
\text { Bronchiectasis [42] } \\
\text { Cystic fibrosis [45] } \\
\text { COPD [43] }\end{array}$ & $\begin{array}{l}\text { No direct evidence } \\
\text { - Use in LAC with caution as oscillations may cause } \\
\text { airway collapse and irritation in some patients } \\
\text { - If used comfortably, may have similar benefits } \\
\text { to OPEP }\end{array}$ \\
\hline HFCWO & $\begin{array}{l}\text { Patient wears an inflatable vest attached to a machine } \\
\text { that creates positive and negative pressure changes } \\
\text { through high-frequency air pulses at a set pressure } \\
\text { and frequency. Displacement of the airway walls } \\
\text { additionally disengages secretions, enhancing airflow } \\
\text { and ciliary beating }\end{array}$ & $\begin{array}{l}\text { Physiological } \\
\text { background [22] } \\
\text { Bronchiectasis [47] } \\
\text { Cystic fibrosis } \\
{[49,50]} \\
\text { COPD [48] }\end{array}$ & $\begin{array}{l}\text { No direct evidence } \\
\text { - Clinical experience suggests this technique must be } \\
\text { combined with FET (with relevant principles } \\
\text { discussed for patients with LAC) }\end{array}$ \\
\hline CPAP & $\begin{array}{l}\text { Creates a "pneumatic splint", helping to prevent } \\
\text { dynamic airways collapse as flow and effort increase }\end{array}$ & Paediatric TBM [51] & $\begin{array}{l}\text { Extrapolation of knowledge from paediatric TBM } \\
\text { although caution required as different conditions } \\
\text { - Clinical expertise/extrapolation of knowledge from } \\
\text { use of PEP/high PEP } \\
\text { - Caution required as underlying pathology may } \\
\text { be different }\end{array}$ \\
\hline
\end{tabular}

ACT: airway clearance technique; ACBT: Active Cycle of Breathing Technique; TEE: thoracic expansion exercise; FET: two forced expiration technique; LAC: large airway collapse; TBM: tracheobronchomalacia; PEP: positive expiratory pressure; OPEP: oscillating expiratory pressure; HFCWO: high-frequency chest wall oscillation; CPAP: continuous positive airway pressure.

\section{Active Cycle of Breathing techniques}

Active Cycle of Breathing (ACBT) consists of breathing control, thoracic expansion exercises and the FET [33]. It can be adapted to individual need, but with each component of the cycle clearly defined (figure 3). Other techniques include Autogenic Drainage or the FET used alone. There is no specific evidence supporting the efficacy of these techniques in LAC patients; however, there is evidence to show ACBT is effective and efficient in the mobilisation and clearance of secretions and improvement in lung function in other respiratory conditions [33] including bronchiectasis [34] and cystic fibrosis [25]. Moreover, they are simple to perform and not associated with any complications/harmful effects [33].

Recommendations for ACBT:

- All patients should be taught ACBT. If ineffective, other techniques may be chosen.

- The length of each phase is flexible and should be adapted to individual patient need and their symptoms (e.g. longer breathing control if breathless).

- Patients with a productive cough should be advised to use this technique regularly, and ideally oncetwice a day between infections but should expect to increase the frequency of use during exacerbations (minimum twice daily).

\section{Positive expiratory pressure}

To assist with airway clearance, positive expiratory pressure (PEP) can be generated in the airways by blowing into a device with a valve that provides resistance to the expiratory flow [35]. "PEP" is a flow-regulated technique that has three effects: to increase lung volume (causing a temporary increase in functional residual capacity (FRC)), to reduce hyperinflation and to improve airway clearance [36]. It is used during tidal breathing to facilitate increased interdependence between alveoli via increased collateral ventilation. This enables air to reach deeper into the lung, specifically the small airways, thus facilitating the movement of air and sputum plugs from the small to the large airways [37]. Evidence in both cystic fibrosis [38] and COPD [39] suggest that it may be useful in these conditions as an alternative technique to 


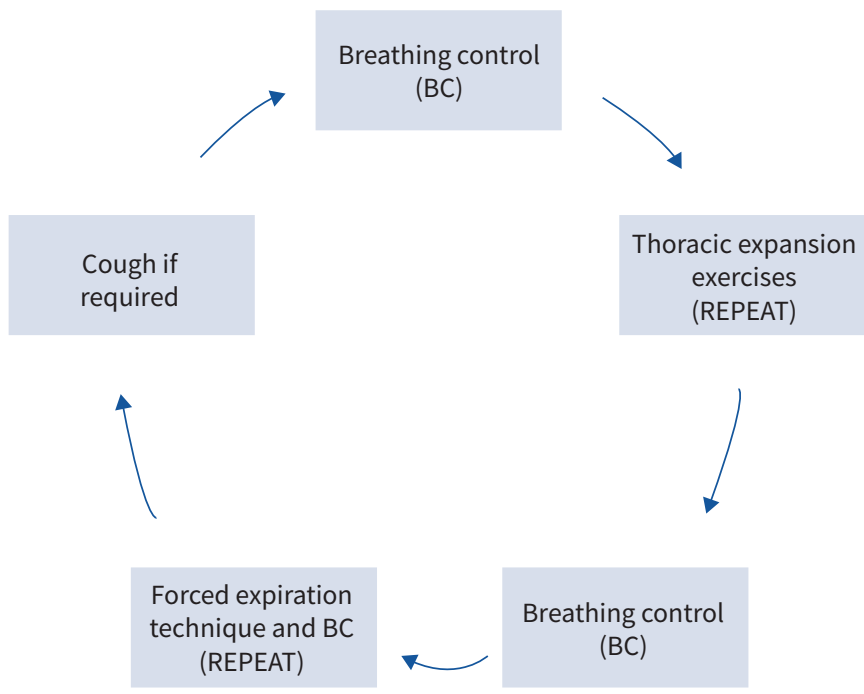

FIGURE 3 The Active Cycle of Breathing Technique.

ACBT. Additionally the use of PEP can ameliorate airflow limitation by splinting open collapsible airways (figure 4) and enabling tidal breathing without encroaching on closing volumes [22], both of which are likely to be especially important in LAC.

In LAC, clinical experience has shown that PEP, delivered via a PEP mask to support ACT (figure 5) for both tidal breathing and with FET, can be used to improve tidal volumes and prevent proximal airway collapse during forced manoeuvres. Caution is required however, as use of this resistance could also limit expiratory airflow if applied incorrectly. SiRITHANGKul et al. [40] studied 40 children (aged below 14 years) with TBM. They performed a technique called "cough spirometry", evaluating cough expiratory flow at the 25-75\% flow point (i.e. determining the effectiveness of cough at mid lung volumes). Using this technique, they evaluated cough with the addition of PEP at 5, 10, 15 and $20 \mathrm{cmH}_{2} \mathrm{O}$ compared with 21 healthy controls. Cough flow increased at 5, 10 and $15 \mathrm{cmH}_{2} \mathrm{O}$, respectively, but decreased at $20 \mathrm{cmH}_{2} \mathrm{O}$. In comparison, in healthy controls, PEP impaired airflow. Thus, it appears that lower pressures of PEP were helpful for augmenting flow. In practice, the assessment of use of PEP in tidal breathing or with the addition of FET and cough requires skilled assessment, in order to ensure the optimal pressure is chosen to minimise the impact of airway collapse and to optimise expiratory airflow and subsequent secretion clearance.

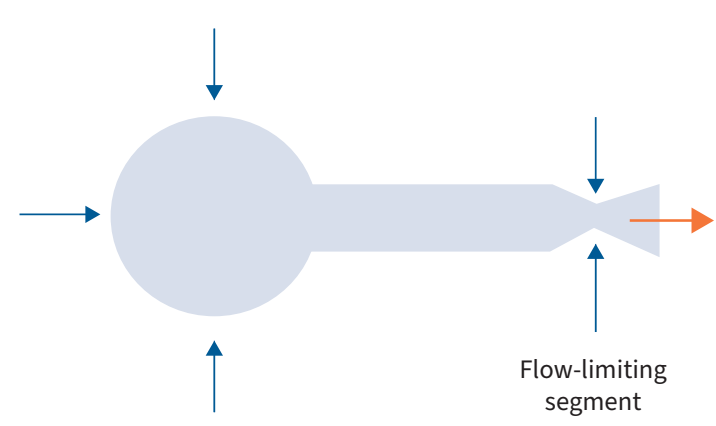

Elevated intrathoracic pressure can compress unstable airways during expiration

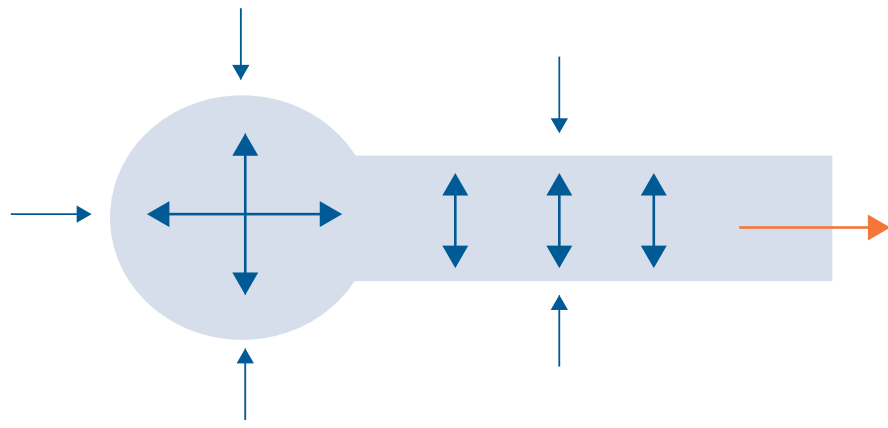

PEP creates back pressure, splints open the airways therefore improving the airflow + /- mucus 
Recommendations for PEP:

- PEP should be considered as a potential ACT for patients with LAC who present with retained secretions and impaired cough effectiveness, despite ACBT and/or FET.

- Use with tidal breathing and/or during FET should be considered.

- Patient preference and tolerability should be considered when choosing an appropriate airway clearance modality.

\section{Oscillatory devices \\ Oscillatory PEP}

Oscillatory PEP (OPEP) is an alternative technique, whereby PEP is generated by blowing out against a variable resistance that also produces an oscillation in flow [34]. Individuals breathe out to expiratory reserve volume enabling a modulation of both pressure and flow.

There are a number of devices that can be used for this, including the Flutter ${ }^{\mathrm{TM}}$, PariOPEP ${ }^{\mathrm{TM}}$, Aerobika ${ }^{\mathrm{TM}}$ and Acapella ${ }^{\mathrm{TM}}$. All OPEP devices aim to help with loosening viscous secretions as they provide a frequency of oscillation within the range necessary to decrease the viscoelasticity and spinnability of mucus, which may provide a benefit for some patients with secretions that are difficult to clear [31]. Most of these devices are closed systems (Aerobika ${ }^{\mathrm{TM}}$ and Acapella ${ }^{\mathrm{TM}}$ ) allowing patients to breathe in through the device, therefore having the added benefit of PEP being maintained throughout the breath [22]. Studies in bronchiectasis suggest the Acapella ${ }^{\mathrm{TM}}$ may improve quality of life in comparison to no ACT [41] and is also safe to use in exacerbations [42]. A recent review in COPD also showed modest evidence that the use of OPEP devices was associated with decreased symptoms of COPD and exacerbations (OR 0.37, 95\% CI 0.19-0.72) [43] and may have a superior effect on length of stay during exacerbations of COPD compared to PEP alone [44]. Additionally evidence in cystic fibrosis suggests that OPEP is not more or less effective than other forms of ACT [45] and may be chosen if a patient prefers this device and resources allow.

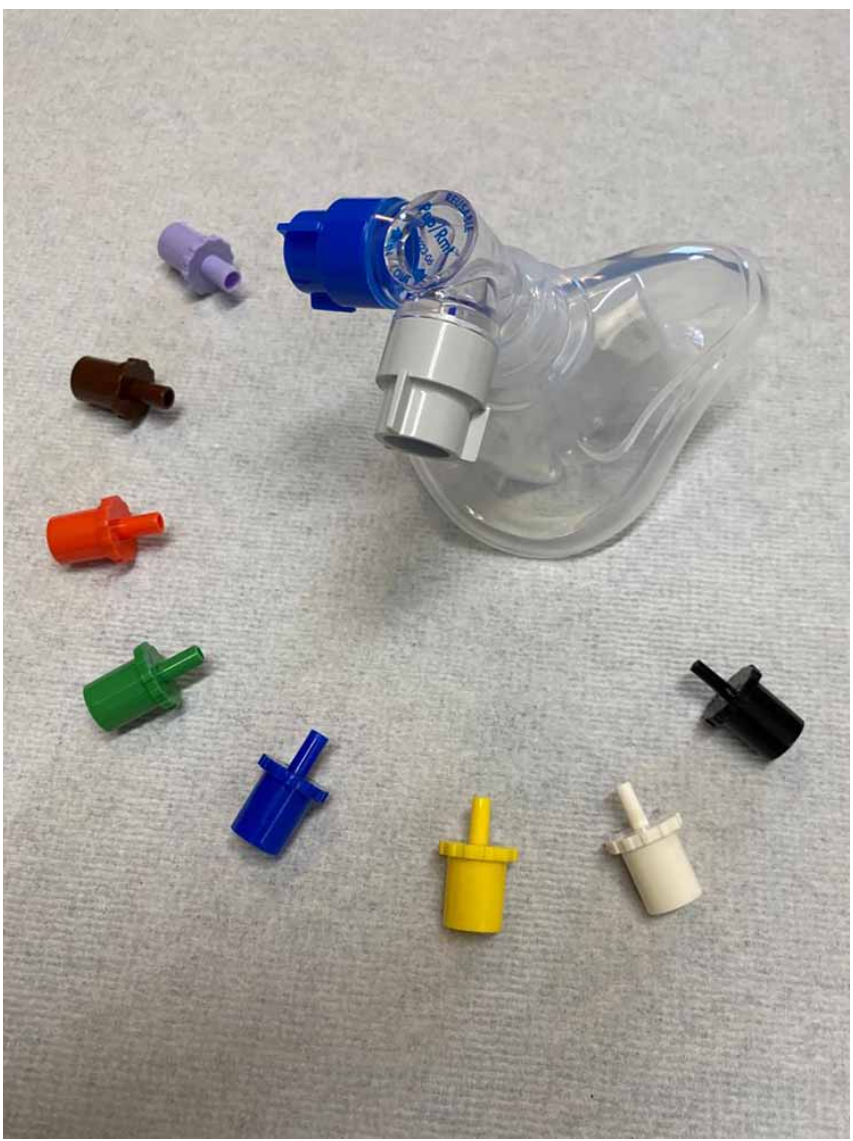


High-frequency chest wall oscillation

High-frequency chest wall oscillation (HFCWO) involves the patient wearing an inflatable vest attached to a machine that creates positive and negative pressure changes through high-frequency air pulses at a set pressure (between 5 and $20 \mathrm{cmH}_{2} \mathrm{O}$ ) and frequency (between 2 and $25 \mathrm{~Hz}$ ). These pressure changes cause short bursts of expiratory air flow of up to $1.6 \mathrm{~L} \cdot \mathrm{s}^{-1}$ within the airway, increasing the air-liquid shear forces during expiration. Additionally, displacement of the airway walls may disengage secretions and enhance the effect of air-liquid flow as well as enhance ciliary beating [46].

There is no evidence specifically in LAC; however, evidence from bronchiectasis [47] and COPD [48] suggests that secretion clearance may be increased while using the device. However, evidence in cystic fibrosis showed an increase in exacerbation frequency when compared with PEP alone [49]. In the UK it is recommended that the individual uses the device alongside regular thoracic expansion exercises and the FET to ensure secretions moved by the device are cleared effectively from the proximal airways [50]. This device is not widely provided in the UK due to the cost (these devices are usually over £8000), whereas in the USA they are often purchased for patients within insurance policies. The choice to use is often based on preference versus cost, as there is lack of evidence on its superiority versus less expensive treatments [46].

Practitioners should be aware that some patients with LAC struggle with oscillatory devices (OPEP and HFCWO), as they may stimulate coughing. The oscillation in flow produced by the devices can increase turbulence within the airways, increasing resistance, and thus further limiting flow. Airflow in the airways already impaired by the floppy proximal airways may be exacerbated further by the oscillations produced.

Recommendations for OPEP:

- Assessment of the impact of the device on coughing and potential increased irritability of airways is important.

- Consider use of devices that utilise a closed system to maintain PEP while providing oscillations (e.g. Acapella $^{\mathrm{TM}}$ and Aerobika ${ }^{\mathrm{TM}}$ ).

\section{CPAP and NIV}

Some patients with LAC may have access to a positive pressure ventilation system and most often this will be a CPAP device prescribed to treat associated sleep disordered breathing. Clinical experience suggests that patients often describe disturbed sleep due to coughing, choking, breathlessness and secretions "getting stuck". The addition of CPAP as an airway clearance adjunct is based on the theory that CPAP may work as a "pneumatic splint", helping to prevent dynamic airways collapse as flow and effort increase $[10,51]$. Physiological increases in flow at FRC arise secondarily to an increase in lung volume with CPAP [52]. This may help in generating higher elastic recoil and increased expiratory flow [53]. Moreover, if intraluminal pressure increases, this may "stiffen" the airways leading to less turbulence from waves and lower resistance, increasing expiratory flow [11].

Evidence from TBM studies in infants/children indicates CPAP improves gas flow at FRC and reduces atelectasis, helping to open closed airways [54]. However, there may be a point (of optimal flow), where the CPAP provides an increase in lung volume, but the individual is not flow-limited during tidal breathing [55]. Clinical experience has shown that NIV (bilevel) may be preferred in some patients (e.g. those requiring higher positive end-expiratory pressure (PEEP) or with coexisting lung disease). In individuals with more severe symptoms that are not optimised by independent breathing techniques or PEP, the addition of CPAP can be helpful.

Patients can be taught to use CPAP in a variety of ways. CPAP can be used prior to airway clearance to optimise ventilation prior to ACT by opening up airways and improving FRC [11]. Additionally, some patients may be taught to use CPAP for tidal breathing (e.g. as they would a PEP device) before removing it to complete FET. In some cases, patients may benefit from use during FET as this could help prevent airways collapse (as previously described). Assessment and training must ensure that CPAP used in this way does not prevent expiratory airflow. Some centres have started to evaluate level of CPAP pressure required to maintain airway patency with expiratory manoeuvres, which may help with selection of pressures [55]. In the absence of this information, we recommend that a physiotherapist look at cough effectiveness (e.g. measuring sputum expectorated), the patient's subjective evaluation of their cough as well as assessment of the cough sound (reduction in stridulous noise and increase in effective cough sounds) having used CPAP.

Prior to using CPAP or adapting pressures, a risk assessment should be completed. This should include a review of potential risk factors including pneumothorax, increased blood pressure and history of pulmonary hypertension. Any concerns should be discussed with the medical team, prior to use. If 
suitable, CPAP should be set on a fixed pressure. Clinical experience to date describes that this is often at higher pressure than any overnight setting due to the need to prevent the collapse in LAC caused by the increased expiratory airflow produced during ACT.

Recommendations for CPAP/NIV:

- CPAP/NIV may be considered in patients with LAC to enhance airway clearance if cough effectiveness is inhibited by dynamic airways collapse. Ensuring that the patient is not flow-limited with CPAP would need consideration with any application.

- Use of CPAP at overnight settings would be a starting point for pressure and selection of a fixed level should be considered. A full risk assessment should be completed before any changes to pressures.

- The effectiveness of CPAP should then be assessed, based on whether secretions are cleared and airway collapse is minimised (assessed by sputum expectorated and audible changes to a patient's subjective report of cough).

- CPAP can be evaluated on bronchoscopy to help select the pressure that minimises collapse on coughing and/or FET.

\section{Mucolytics}

As commonly found in other respiratory diseases, secretions may become thick and sticky and difficult to remove, particularly during chest infections. In this instance, it may be appropriate to consider the use of nebulised mucolytics, to assist with secretion removal. Humidification may reduce sputum viscosity, improve airway hydration and decrease airway irritability [24]. Mucolytics could be used pre, post and during airway clearance.

Hypertonic saline (HTS), for example, may increase airway surface liquid, alter sputum rheology by breaking ionic bonds and increase ionic concentration within the mucin gel in the sputum [56]. It may also increase cough frequency, which in other conditions could enhance clearance; however, in LAC, a vigorous and sustained increase in coughing may be counter-productive. Guidelines in bronchiectasis suggest that a trial of normal saline (NS) or HTS to facilitate clearance may be appropriate if other strategies have been unsuccessful [30]. Preliminary data in 69 patients with LAC nebulising HTS delivered by mouthpiece PEP device showed a reduction in annualised exacerbation rate from a mean (min-max) of 4 (0-28) compared to $2.8(0-7)$ [57]. Preliminary data from our centre also suggest a possible role of nebulised $\mathrm{N}$-acetyl cysteine. This is used in patients who might benefit from a mucolytic but who cannot tolerate HTS. Anecdotally, some do very well and attribute their sustained reduction in respiratory infections to using this within their airway clearance regime.

Recommendations for mucolytics:

- A trial of mucoactive medications (NS or HTS) may be appropriate for patients with thick and sticky secretions whose ACT is not optimised. Such medication should be trialled for tolerability and safety with a patient prior to commencement and reviewed for effectiveness.

\section{B: Breathing (breathlessness and breathing pattern)}

Patients with LAC often report pervasive breathlessness, and in most cases this will be multifactorial in nature [14]. Indeed, in many patients there will be a contribution from obstructive components of lung disease especially during exercise (see below), but additionally patients with LAC often complain of expiratory and sometimes inspiratory sensations of airway closure within the larger airway, especially with forced and/or expiratory manoeuvres. It is becoming increasingly well recognised that there is often an overlap between LAC and laryngeal dysfunction [58]. Expiratory laryngeal narrowing as well as mechanisms including pursed lip breathing (PLB) may be functionally helpful to create intrinsic positive end-expiratory pressure and splinting open of the airways in expiration, which has also been shown to be present in patients with moderate/severe COPD [59].

Unfortunately, there is also a risk that such adaptations could also lead to breathing pattern dysfunction (BPD) and/or inducible laryngeal obstruction at the most extreme, through increased tension in the structures around the larynx [59]. Ineffective coughing, discomfort in the large airways and changes to breathing pattern (mouth breathing, gasping, apical breaths) could also increase the sensitivity of the larynx and may increase cough frequency as well as increase the sensation of breathlessness.

Assessment of breathlessness and breathing pattern

The evaluation of breathing should look broadly at breathlessness but also more specifically at breathing pattern. Breathlessness symptoms and triggers should be recorded as described by the patient, including its nature, what makes it worse, how the patient manages and improves it. This should occur concurrently 
with a physical assessment evaluating "work of breathing” and breathing pattern. Breathing pattern assessment should include evaluation of respiratory rate, upper or lower chest breathing, whether it is regular or erratic, and evaluation of inspiratory and expiratory flow. Use of the Brompton Breathing Pattern Assessment Tool (BPAT) may be helpful to organise this assessment and improve recognition of those with BPD [29]. Symptoms within the upper airway should also be assessed to ensure any laryngeal components are considered.

\section{Treatment}

Patients who are breathless may benefit from breathing pattern re-education to optimise nasal breathing at rest and reduce thoracic/upper chest breathing. Reducing the turbulence of flow into the upper airway and during expiration may minimise symptoms of collapse, as well as reduce irritation and dehydration. It is also important that patients are given strategies to ensure they are not over-using their upper airway when creation of intrinsic PEEP is required. Modification of this and encouragement to use PLB may be important as well as education in recognising increased tension in the upper airway during inspiration [60].

For some patients, breathlessness on exertion is so severe that breathing modifications are not enough. This is when using positive pressure support on exertion through CPAP may be useful (see below).

\section{C: Capacity for exercise}

Impaired exercise tolerance is a key and pervasive feature for many patients with severe LAC [61]. Exercise increases tidal volume and minute ventilation, resulting in an increase in driving pressure $\left(P_{\text {alv }}\right)$ and $P_{\mathrm{pl}}$ increasing turbulent flow and resistance. This may lead to flow limitation and possible early airway closure which increases expiratory airway collapse [15, 62] and increases the sensation of breathlessness. The unpredictability of pathophysiology and degree of functional limitation will be heterogeneous within each lung/segment and within each patient. Predominant symptoms during exercise are breathlessness and expiratory wheeze, often with no changes on flow volume loops at rest or during exercise.

\section{Assessment}

Patients with LAC should be evaluated with a functional exercise assessment (e.g. 6-min walk test, 6MWT) to formally evaluate functional ability [63]. During this assessment the patient's physiological parameters should be measured (heart rate, oxygen saturation measured by pulse oximetry $\left(S_{\mathrm{pO}_{2}}\right)$, Borg rating of perceived exertion (RPE)) as well as evaluation of the patient's breathing pattern. Moreover, assessment of airway collapse should be made through listening to the patient's breathing, especially on expiration. Other symptoms to observe include upper airway symptoms and/or coughing [62].

\section{Treatment}

Techniques to support exercise tolerance should optimise expiratory airflow in an otherwise narrowed lumen. Initially the focus should be on increasing awareness of the impact of increased flow, especially on inspiration. This may be by encouragement of nasal breathing to promote laminar flow, and/or with the addition of PLB to reduce expiratory flow by providing a resistance to breathe out against, as previously described. This may also reduce the turbulent flow upstream. Evidence comes from a small study where patients were taught either PLB, oral PEP (PEP delivered through a device with a resistor fitted and worn in the mouth) and nasal PEP (as oral, but worn at the nose) [64]. Continuous upper airway monitoring was used to assess airway pressure. The results showed some evidence of reduced breathlessness and increased function with use of airway splinting by these different techniques.

\section{Ambulatory CPAP}

The use of CPAP during ambulation may ameliorate early airway collapse and improve exercise capacity. KaltsaKAs et al. [65] trialled 19 patients with LAC using CPAP at pressures of 4, 7 and $10 \mathrm{cmH}_{2} \mathrm{O}$ or a sham. Patients then underwent repeat 6MWT on sham or active CPAP in a random order. Neural respiratory drive index (NRDI) was also measured. 6MWT distance on optimal CPAP was increased compared to self-ventilation and sham CPAP. Treatment effect between sham and optimal CPAP was $31 \pm 39$ m (95\% CI 13 to $50 \mathrm{~m}$ ). Respiratory muscles were more unloaded in the CPAP group, as measured by NRDI. Additional evidence supports the utility of CPAP in this group: 27 patients with LAC had a 6MWT on air versus CPAP [66]. The distance achieved with 6MWT improved significantly with CPAP (by median (range) $32(8-53) \mathrm{m})$ compared to room air $(\mathrm{p}=0.003)$. The mean PEEP used was $13 \pm 4 \mathrm{cmH}_{2} \mathrm{O}$, which is higher than most average overnight settings. Clinical use at our centre suggests PEEP should be set at resting settings or just above. This may be titrated to ensure LAC symptoms are optimised without increasing the work of breathing (likely between 10 and $14 \mathrm{cmH}_{2} \mathrm{O}$ ). Additionally, a fixed pressure is advised for use with exercise and may enhance the effectiveness of pulmonary 
rehabilitation in this population. For a suggested assessment protocol, please see Ambulatory CPAP assessment protocol.

Recommendations:

- All patients should be encouraged to participate in regular physical activity and should be educated to meet the minimum guidelines for physical activity provided by the American College of Sports Medicine.

- Physical training programmes should include aerobic and resistance training, which have been shown to demonstrate clinically meaningful improvements in whole body endurance and strength as per pulmonary rehabilitation guidelines (e.g. British Thoracic Society Pulmonary Rehabilitation Guidelines).

- Patients whose breathlessness affects activities of daily living (MRC >3) should be referred to pulmonary rehabilitation, as extrapolated from other conditions including COPD, bronchiectasis and asthma.

- Patients should be taught how to manage their exercise/activity symptoms with control of flow, nasal breathing, breathing control and PLB techniques.

- In patients who are symptomatic of LAC during exercise (cough, stridor, breathlessness, reduced exercise capacity), the use of NIV/CPAP should be considered and used during exercise training and/or pulmonary rehabilitation.

Ambulatory CPAP Assessment Protocol:

- A full risk assessment should be completed prior to any commencement or change in level of positive pressure in accordance with local service guidelines.

- A 6MWT should be completed on air and on CPAP to assess for any increase in exercise capacity and/ or symptom reduction.

- PEEP should be set on a fixed pressure at resting settings or just above with titration as required to optimise comfort.

- If patients are unable to complete a 6MWT, a repeated sit-to-stand test for 1 min should be completed [67].

- Subjective measures of perceived exertion, breathlessness and fatigue should be used to assess symptoms during exercise.

- In patients with severe symptoms or already utilising NIV, this should be used instead of CPAP.

\section{Limitations}

A key limitation to this review is the paucity of published evidence to support physiotherapy interventions in the management of LAC. This review provides a timely opportunity to explore the physiological principles underpinning techniques commonly used by physiotherapists, in the context of the clinical challenges of this condition. By extrapolating evidence from other respiratory conditions, and drawing on associated clinical expertise, this has enabled us to rationalise the value of the interventions being delivered by physiotherapists to support the conservative management of patients with LAC and highlights the need to develop protocols to evaluate the physiological principles discussed.

\section{Conclusion}

In our experience, physiotherapists play a key role in the assessment and management of individuals with LAC. The ABC model, described herein, provides a therapy framework for LAC, outlining the importance of evaluating Airways, Breathing and Capacity for exercise, as part of a comprehensive management strategy. Despite this, there remains a paucity of high-quality research in this area to help inform the best choice of treatment, and this is a clear limitation to the review. In the absence of these data, assimilation of clinical expertise and extrapolation from literature addressing other similar conditions provides therapists with the best way to assess and treat the pathophysiological impairments of LAC. It remains a priority area for future studies to improve treatment selection in this important condition.

Provenance: Submitted article, peer reviewed.

Acknowledgements: Claire Pyatt is a patient with large airway collapse and gave permission for the audio recording of her cough to be shared.

Conflict of interest: None declared.

Support statement: L.J.F. Grillo is a current Pre-Clinical Academic Fellow with the National Institute of Health Research (NIHR) UK. 
References

1 Mitropoulos A, Song W-J, Almaghlouth F, et al. Detection and diagnosis of large airway collapse: a systematic review. ERJ Open Res 2021; 7: 3.

2 Leong P, Bardin PG, Lau KK. What's in a name? Expiratory tracheal narrowing in adults explained. Clin Radiol 2013; 68: 1268-1275.

3 Diaz Milian R, Foley E, Bauer M, et al. Expiratory central airway collapse in adults: anesthetic implications (part 1). J Cardiothorac Vasc Anesth 2019; 33: 2546-2554.

4 Kheir F, Majid A. Tracheobronchomalacia and excessive dynamic airway collapse: medical and surgical treatment. Semin Respir Crit Care Med 2018; 39: 667-673.

5 Buitrago $\mathrm{DH}$, Wilson JL, Parikh M, et al. Current concepts in severe adult tracheobronchomalacia: evaluation and treatment. J Thoracic Dis 2017; 9: 57-66.

6 Jokinen K, Palva T, Sutinen S, et al. Acquired tracheobronchomalacia. Ann Clin Res 1977; 9: 52-57.

7 Joosten S, MacDonald M, Lau KK, et al. Excessive dynamic airway collapse co-morbid with COPD diagnosed using 320-slice dynamic CT scanning technology. Thorax 2012; 67: 95-96.

8 Dal Negro RW, Tognella S, Guerriero M, et al. Prevalence of tracheobronchomalacia and excessive dynamic airway collapse in bronchial asthma of different severity. Multidiscip Respir Med 2013; 8: 32.

9 Adliff M, Ngato D, Keshavjee S, et al. Treatment of diffuse tracheomalacia secondary to relapsing polychondritis with continuous positive airway pressure. Chest 1997; 112: 1701-1704.

10 Patout M, Mylott L, Kent R, et al. Trial of portable continuous positive airway pressure for the management of tracheobronchomalacia. Am J Respir Crit Care Med 2016; $193: 57$.

11 Ferguson GT, Benoist J. Nasal continuous positive airway pressure in the treatment of tracheobronchomalacia. Am Rev Respir Dis 1993; 147: 457-461.

12 Majid A, Alape D, Kheir F. Short-term use of uncovered self-expanding metallic airway stents for severe expiratory central airway collapse. Respiration 2016; 92: 389-396.

13 Boiselle PM, O'Donnell CR, Bankier A, et al. Tracheal collapsibility in healthy volunteers during forced expiration: assessment with multidetector CT. Radiology 2009; 252: 255-262.

14 Murgu S, Colt H. Tracheobronchomalacia and excessive dynamic airway collapse. Clin Chest Med 2013; 34: 527-555.

15 Biswas A, Jantz MA, Sriram PS, et al. Tracheobronchomalacia. Dis Mon 2017; 63: 287-302.

16 Mead J, Turner JM, Macklem PT, et al. Significance of the relationship between lung recoil and maximum expiratory flow. J Appl Physiol 1967; 22: 95-108.

17 Litmanovich C D, O'Donnell R, Bankier AA, et al. Bronchial collapsibility at forced expiration in healthy volunteers: assessment with multidetector CT. Radiology 2010; 257: 560-567.

18 Dawson SV, Elliott EA. Wave speed limitation on expiratory flow: a unifying concept. J Appl Physiol Respir Environ Exercise Physiol 1977; 43: 498-515.

19 Handa H, Miyazawa T, Murgu SD, et al. Novel multimodality imaging and physiologic assessments clarify choke-point physiology and airway wall structure in expiratory central airway collapse. Respir Care 2012; 57: 634-641.

20 van der Schans CP, Postma DS, Koëter GH, et al. Physiotherapy and bronchial mucus transport. Eur Respir J 1999; 13: 1477-1486.

21 Cole P. The damaging role of bacteria in chronic lung infection. J Antimicrob Chemother 1997; 40: 5-10.

22 Mcllwaine M, Bradley J, Elborn JS, et al. Personalising airway clearance in chronic lung disease. Eur Respir Rev 2017; 26: 160086

23 Kim CS, Iglesias AJ, Sackner MA. Mucus clearance by two-phase gas-liquid flow mechanism: asymmetric periodic flow model. J Appl Physiol (1985) 1987; 62: 959-971.

24 Conway JH. The effects of humidification for patients with chronic airways disease. Physiotherapy (United Kingdom) 1992; 78: 97-101.

25 Main E, Grillo L, Rand S. Airway clearance strategies in cystic fibrosis and non-cystic fibrosis bronchiectasis. Semin Respir Crit Care Med 2015; 36: 251-266.

26 Bott J, Blumenthal S, Buxton M, et al. Guidelines for the physiotherapy management of the adult, medical, spontaneously breathing patient. Thorax 2009; 64: Suppl 1, i1-i51.

27 Chamberlin Mitchell SA, Garrod R, Clark L, et al. Physiotherapy, and speech and language therapy intervention for patients with refractory chronic cough: a multicentre randomised control trial. Thorax 2017; 72: $129-136$

28 Morice AH, Millqvist E, Bieksiene K, et al. ERS guidelines on the diagnosis and treatment of chronic cough in adults and children. Eur Respir J 2020; 55: 1901136.

29 Todd S, Walsted ES, Grillo L, et al. Novel assessment tool to detect breathing pattern disorder in patients with refractory asthma. Respirology 2018; 23: 284-290.

30 Hill AT, Sullivan A, Chalmers J, et al. British Thoracic Society guideline for bronchiectasis in adults. Thorax 2019; 74: Suppl. 1, 1-69. 
31 Flude LJ, Agent P, Bilton D. Chest physiotherapy techniques in bronchiectasis. Clin Chest Med 2012; 33: 351-361.

32 Pryor JA. Physiotherapy for airway clearance in adults. Eur Respir J 1999; 14: 1418-1424.

33 Lewis LK, Williams MT, Olds TS. The active cycle of breathing technique: a systematic review and meta-analysis. Respir Med 2012; 106: 155-172.

34 Eaton T, Young P, Zeng I, et al. A randomized evaluation of the acute efficacy, acceptability and tolerability of Flutter and active cycle of breathing with and without postural drainage in non-cystic fibrosis bronchiectasis. Chron Respir Dis 2007; 4: 23-30.

35 Olsén FM, Lannefors LL, Westerdahl E. Positive expiratory pressure: common clinical applications and physiological effects. Respir Med 2015; 109: 297-307.

36 Fagevik Olsén $\mathrm{M}$, Olofsson $\mathrm{P}$, Frejd $\mathrm{P}$, et al. Technical aspects of devices and equipment for positive expiratory pressure with and without oscillation. Respir Care 2021; 66: 862-877.

37 Falk M, Kelstrup M, Andersen J, et al. Improving the ketchup bottle method with positive expiratory pressure, (PEP), in cystic fibrosis. Eur J Respir Dis 1984; 65: 423-432.

38 Elkins M, van der Schans C, Jones A. Positive expiratory pressure physiotherapy for airway clearance in people with cystic fibrosis. Cochrane Database Syst Rev 2006; 2: CD003147.

39 Nicolini A, Mascardi V, Grecchi B, et al. Comparison of effectiveness of temporary positive expiratory pressure versus oscillatory positive expiratory pressure in severe COPD patients. Clin Respir J 2018; 12: 1274-1282.

40 Sirithangkul S, Ranganathan S, Robinson PJ, et al. Positive expiratory pressure to enhance cough effectiveness in tracheomalacia. J Med Assoc Thailand 2010; 93: Suppl. 6, 112-118.

41 Murray MP, Pentland JL, Hill AT. A randomised crossover trial of chest physiotherapy in non-cystic fibrosis bronchiectasis. Eur Respir J 2009; 34: 1086-1092.

42 Patterson JE, Hewitt O, Kent L. Acapella versus 'usual airway clearance' during acute exacerbation in bronchiectasis: a randomized crossover trial. Chron Respir Dis 2007; 4: 67-74.

43 Alghamdi SA, Barker RE, Alsulayyim AS, et al. Use of oscillatory positive expiratory pressure (OPEP) devices to augment sputum clearance in COPD: a systematic review and meta-analysis. Thorax 2020; 75: 855-863.

44 Milan S, Bondalapati P, Megally M, et al. Positive expiratory pressure therapy with and without oscillation and hospital length of stay for acute exacerbation of chronic obstructive pulmonary disease. Int J Chron Obstruct Pulmon Dis 2019; 14: 2553-2561.

45 Morrison L, Milroy S. Oscillating devices for airway clearance in people with cystic fibrosis. Cochrane Database Syst Rev 2017; 5: CD006842.

46 Bradley JM. High frequency chest wall oscillation in cystic fibrosis. Thorax 2010; 65: 189-190.

47 Nicolini A, Cardini F, Landucci N, et al. Effectiveness of treatment with high-frequency chest wall oscillation in patients with bronchiectasis. BMC Pulm Med 2013; 13: 21.

48 Nicolini A, Grecchi B, Ferrari-Bravo M, et al. Safety and effectiveness of the high-frequency chest wall oscillation vs intrapulmonary percussive ventilation in patients with severe COPD. Int J Chron Obstruct Pulmon Dis 2018; 13: 617-625.

49 Mcilwaine MP, Alarie N, Davidson GF, et al. Long-term multicentre randomised controlled study of high frequency chest wall oscillation versus positive expiratory pressure mask in cystic fibrosis. Thorax 2013; 68 : 746-751.

50 Osman LP, Roughton M, Hodson ME, et al. Short-term comparative study of high frequency chest wall oscillation and European airway clearance techniques in patients with cystic fibrosis. Thorax 2010; 65: 196-200.

51 Panitch HB, Allen JL, Alpert BE, et al. Effects of CPAP on lung mechanics in infants with acquired tracheobronchomalacia. Am J Respir Crit Care Med 1994; 150: 1341-1346.

52 López-Padilla D, García-Luján R, Maestu LP, et al. Tracheobronchomalacia treatment: how far have we come? J Thoracic Dis 2016; 8: 3490-3493.

53 Lima E, Nakamura M, Genta P, et al. Improving airways patency and ventilation through optimal positive pressure identified by noninvasive mechanical ventilation titration in Mounier-Kuhn syndrome: protocol for an interventional, open-label, single-arm clinical trial. JMIR Res Protoc 2020; 9: e14786.

54 Wallis C, Alexopoulou E, Antón-Pacheco JL, et al. ERS statement on tracheomalacia and bronchomalacia in children. Eur Respir J 2019; 54: 1900382.

55 Loring SH, O'Donnell CR, Feller-Kopman DJ, et al. Central airway mechanics and flow limitation in acquired tracheobronchomalacia. Chest 2007; 131: 1118-1124.

56 Elkins MR, Bye PTP. Mechanisms and applications of hypertonic saline. J R Soc Med Suppl 2011; 104: 2.

57 Sheehan R, Abu-El-Hawa N, Tavernier G, et al. P139 Impact of nebulised hypertonic saline using positive expiratory pressure on exacerbations in adults with large airway collapse with symptoms (LACS). Thorax 2018; 73: Suppl. 4, 177.

58 Weinstein DJ, Hull JE, Ritchie BL, et al. Exercise-associated excessive dynamic airway collapse in military personnel. Ann Am Thorac Soc 2016; 13: 1476-1482. 
59 Rendo M, Sjulin T, Morris M, et al. Upper airway wheezing: inducible laryngeal obstruction vs excessive dynamic airway collapse. Chest 2017; 152: 100827.

60 Leong P, Tran A, Rangaswamy J, et al. Expiratory central airway collapse in stable COPD and during exacerbations. Respir Res 2017; 18: 163.

61 Murgu SD, Colt HG. Tracheobronchomalacia and excessive dynamic airway collapse. Respirology 2006; 11: 388-406.

62 Carden KA, Boiselle PM, Waltz DA, et al. Tracheomalacia and tracheobronchomalacia in children and adults: an in-depth review. Chest 2005; 127: 984-1005.

63 ATS Committee on Proficiency Standards for Clinical Pulmonary Function Laboratories. ATS statement: guidelines for the six-minute walk test. Am J Respir Crit Care Med 2002; 166: 111-117.

64 Zafar MA, Mulhall AM, Eschenbacher W, et al. Manometry Optimized Positive Expiratory Pressure (MOPEP) in Excessive Dynamic Airway Collapse (EDAC). Respir Med 2017; 131: 179-183.

65 Kaltsakas G, Patout M, Arbane G, et al. Effect of ambulatory continuous positive airway pressure on neural respiratory drive and functional capacity in excessive dynamic airway collapse. Eur Respir J 2018; 52: Suppl. 62, PA3362.

66 Razakamanantsoa L, Chahine B, Goument L, et al. Portable CPAP benefits on exercise capacity in patients with excessive dynamic airway collapse (EDAC). Eur Respir J 2019; 54: Suppl. 63, PA672.

67 Reychler G, Boucard E, Peran L, et al. One minute sit-to-stand test is an alternative to 6MWT to measure functional exercise performance in COPD patients. Clin Respir J 2018; 12: 1247-1256. 